\title{
Two-Dimensional Versus Three-Dimensional Laparoscopic Systems in Urology: A Systematic Review and Meta-Analysis
}

\author{
Najib Isse Dirie, MD, Qing Wang, MD, and Shaogang Wang, PhD
}

\begin{abstract}
Background: Laparoscopy is widely used in the urological field. This systematic review and a meta-analysis were conducted to assess the clinical and surgical efficacy of the three-dimensional (3D) laparoscopic system in comparison with two-dimensional (2D) laparoscopy for treatment of different urological conditions.

Methods: Following guidelines of Preferred Reporting Items for Systematic Reviews and Meta-Analyses, a systematic literature search in Web of Science, PubMed, Cochrane Library, and EMBase was carried out to identify relevant studies published up to May 2018. Articles published in the English language of both randomized and observational studies comparing 3D and 2D laparoscopic systems in urological surgeries were included. Level of evidence and quality assessments of all included studies were conducted. Interested data were extracted for comparison and meta-analysis.

Results: Our literature search generated 17 studies comparing 3D and 2D laparoscopic systems in different urological surgeries. Of these, 13 studies containing 548 and 449 patients operated on with 2D and 3D laparoscopic systems, respectively, were included for meta-analysis. These 13 studies were divided into three groups according to surgical type. Group 1: Partial nephrectomy (PN); operative time $(p=0.19)$, estimated blood loss (EBL) $(p=0.51)$, dissecting time $(p=0.58)$, and suturing time $(p=0.28)$ were not statistically significant between 2D and 3D laparoscopic systems. However, warm ischemia time during PN was significantly shorter during 3D laparoscopy $(p<0.00001)$. Group 2: Pyeloplasty; this procedure showed no significant difference between the two systems. Group 3: Radical prostatectomy (RP); shorter operative time $(p<0.0001)$ and lower EBL $(p=0.001)$ were associated with the 3D laparoscopic system.

Conclusion: Three-dimensional laparoscopy mainly improves the depth of perception, leading to better visibility, which is important for some complex urological surgeries such as PN, pyeloplasty, and RP. Based on our findings, 3D laparoscopy seems to provide better clinical and surgical outcomes in some urological procedures compared with conventional 2D laparoscopy.
\end{abstract}

Keywords: three-dimensional laparoscopy, two-dimensional laparoscopy, comparison, urological surgeries, meta-analysis

\section{Introduction}

$\mathbf{C}$ Linical apPlication of LAPAROSCOPIC surgery started in the early 1980s. ${ }^{1}$ Since that time, laparoscopy became the preferred choice for many surgeons around the world for its surgical and clinical benefits over traditional open surgery. These benefits include minimized surgical trauma, faster recovery time, reduced postoperative analgesic use, and shorter length of hospital stay. ${ }^{2,3}$ Another attractive ad- vantage of laparoscopic surgery is the cinematic scenery and recordable surgical videos that revolutionized the learning environment in operating rooms and classrooms, as well as presentations during medical conferences. These characteristics together made laparoscopic surgery one of the most significant milestones of the 20th century in the field of surgery. Nevertheless, laparoscopy has some technical and ergonomic challenges mainly when performing intracorporeal laparoscopic sutures. 
There are three main challenges in laparoscopy: (1) the limited range of motion of laparoscopic instruments, (2) poor depth of perception in the two-dimensional (2D) laparoscopic imaging system, and (3) longer learning curve. To tackle these problems, new technologies have been developed such as laparoscopic robotized needle holders that can mimic the surgeon's hand movement ${ }^{4}$; this instrument has simplified intracorporeal suturing difficulties. Other advances include the three-dimensional (3D) laparoscopic imaging system. ${ }^{5}$ Together, these two technologies have improved intracorporeal dissection, suturing quality, ergonomics, and depth of perception during laparoscopic surgery.

Another significant milestone in the field of minimally invasive surgeries is development of the da Vinci robotic system (Intuitive Surgical, Inc., Sunnyvale, CA). Compared with conventional laparoscopy, da Vinci robotics provides better ergonomics and an excellent robotic arm (EndoWrist) that can rotate as much as $7^{\circ}$ and is able to eliminate human hand tremors in addition to its impeded 3D imaging technology. However, a significant limitation of this system is the higher cost to purchase and maintain it. The cost of a da Vinci robotic machine ranges between 1 and 2.3 million dollars depending on the version and its configurations with an additional 180,000 dollars annually for maintenance. In comparison, the 3D laparoscopic system costs around 250,000 dollars plus 25,000 dollars of annual costs. ${ }^{6}$ The above information clearly shows the economic benefits of the 3D laparoscopic system over da Vinci robotics.

The 2D laparoscopic system consists of a single camera (monoscopic), which is the primary reason why this system lacks depth of perception. On the other hand, 3D laparoscopy contains two side-by-side cameras (stereoscopic). Images from these two different cameras pass through an eyeglass in which each eyepiece corresponds to one camera. Finally, images are filtered and received as one; this leads to an increase of depth of perception. ${ }^{7}$ Increased depth of perception is associated with higher accuracy and speed in performance, as well as a shorter learning curve. ${ }^{8}$

Majority of the studies comparing 3D and 2D laparoscopic surgeries focused on novices, experts, residents, and students using training boxes, human cadavers, or animal models. ${ }^{9-11}$ However, there are some clinical trials and comparative observational studies comparing the efficacy, safety, clinical, and surgical outcomes between 2D and 3D laparoscopic systems. ${ }^{12,13}$

Although considerable benefits were associated with the 3D laparoscopic imaging system over conventional 2D laparoscopy, the 3D laparoscopic system is not yet universally adopted. Based on the existing clinical literature, we conducted this systematic review and meta-analysis to assess the safety, efficacy, and clinical outcomes of 3D vs 2D laparoscopic imaging systems during different urological surgeries.

\section{Methods}

\section{Search strategy}

Two separate reviewers performed a systematic search in PubMed, Web of Science, Cochrane Library, and EMBase. We used the following search terms: "three-dimensional laparoscopic" or "3D laparoscopic" and "urology" or "adrenalectomy" or "nephrectomy" or "pyeloplasty" or "cystectomy" or "prostatectomy" to identify relevant studies published up to May 2018. We followed guidelines of Preferred Reporting Items for Systematic Reviews and Meta-Analyses. ${ }^{14}$

\section{Selection criteria}

Inclusion criteria.

- Articles published in the English language or at least their abstract written in the English language.

- Studies comparing 3D and 2D laparoscopic systems in urological surgeries only.

- Both randomized and observational studies.

- Both in adults and children.

\section{Exclusion criteria.}

- Studies that are not reporting patients, but instead comparing urology experts, residents, and students utilizing laparoscopic training boxes, human cadavers, or animal models.

- Studies addressing 3D laparoscopy only and not comparing it with the 2D laparoscopic system.

- Studies comparing 3D and 2D laparoscopic systems in nonurological surgeries.

- Abstract articles for academic conferences without the full article.

\section{Data extraction}

Two reviewers performed data extraction and quality assessment of all the included studies. The following information was extracted from each included study: first author's name, publication year, 3D equipment system, surgical type, number of participants, age, gender, estimated blood loss (EBL), and operative time. We also extracted some specific clinical and surgical outcomes of interest from each surgical type. For instance, urethrovesical anastomosis (UVA) time, positive surgical margins (PSMs), continence recovery, and hospital stay data were extracted from the studies addressing radical prostatectomy (RP), while dissecting time, suturing time, and warm ischemia time (WIT) were obtained from articles reporting partial nephrectomy (PN) for comparison and metaanalysis. Finally, we divided all the included studies according to surgical type for a precise and accurate comparison.

\section{Quality assessment}

We used criteria provided by the Oxford Centre for Evidence-Based Medicine to rate the level of evidence for each study. The Newcastle-Ottawa Scale was used to assess the methodological quality of studies. Two reviewers performed the procedure, respectively. Disagreements were resolved through discussions. If disagreement persisted, a third investigator would participate in the discussion until a consensus was reached.

\section{Statistical analysis}

This meta-analysis compares the efficacy, safety, and overall clinical outcomes of 3D vs 2D laparoscopic systems for different urological surgeries. Review Manager software (RevMan v.5.3; Cochrane Collaboration, Oxford, United Kingdom) was used to analyze the studies in this meta-analysis for comparison. Odds ratio (OR) and mean difference (MD) were used to evaluate dichotomous and continuous variables, respectively. The statistical calculation reported by Hozo and colleagues $^{15}$ was used (when continuous data are reported as means and ranges) to calculate the standard deviation. Results 
are shown with $95 \%$ confidence interval (CI). Heterogeneity of the studies was assessed using the chi-square test. A $p$-value $<0.05$ was considered statistically significant.

\section{Results}

\section{Study characteristics}

Our literature search generated 17 studies (4 randomized and 13 observational) comparing 3D and 2D laparoscopic systems during different urological surgeries. ${ }^{16-32}$ These studies contained 630 and 520 patients who were operated on with 2D and 3D laparoscopic systems, respectively. Author's name, publication year, study design, study characteristics, and quality assessment of all the studies are summarized in Table 1. Of these, four studies ${ }^{17,20,23,27}$ were excluded from the meta-analysis (one adrenalectomy study, one ureterolithotomy study, and one cystectomy study were excluded because there were no similar studies to compare with since we designed our study to compare studies addressing similar procedures, and the fourth study about pyeloplasty was excluded due to lack of a standard deviation parameter for metaanalysis comparison). The remaining 13 studies (3 randomized and 10 observational) containing 548 patients operated on with a 2D laparoscopic system and 449 patients operated on with a 3D laparoscopic system were divided into three groups according to surgical type. Meta-analysis results of these 13 studies are shown in Table 2. Viking 3D HD (United States of America), Karl Storz 3D (Germany), and Olympus 3D (Japan) were the 3D laparoscopic systems used in the studies we reviewed. Flow chart diagram illustrating our search and selection strategy is shown in Figure 1.

\section{Quality assessment}

The evidence levels of studies by Ruan and colleagues ${ }^{25}$ and Kinoshita and colleagues ${ }^{21}$ were rated Level 2, while the rest were Level 3 based on the Oxford level of evidence criteria. The studies by Aykan et al. ${ }^{18}$ and Kinoshita and colleagues ${ }^{21}$ were scored nine stars according to the Newcastle-Ottawa Scale, which was considered to be of high methodological quality. The remaining studies were scored eight stars each.

\section{Meta-analysis}

Partial nephrectomy. For PN, four studies comparing 2D and 3D laparoscopic systems comprising 193 vs 147 patients, respectively, were included. ${ }^{22,25,26,32}$ Using a random effect model, results showed no statistical difference between the two groups on operative time (MD: 9.56; 95\% CI: -4.60 to $23.73 ; p=0.19$ ), EBL (MD: $-12.76 ; 95 \% \mathrm{CI}:-50.56$ to $25.04 ; p=0.51$ ), dissecting time (MD: $0.88 ; 95 \% \mathrm{CI}:-4.02$ to $2.25 ; p=0.58$ ), and suturing time (MD: $2.17 ; 95 \% \mathrm{CI}:-2.19$ to $7.16 ; p=0.28$ ). Warm ischemia time was significantly shorter in the 3D laparoscopic imaging system group compared with the 2D laparoscopic group (MD: $2.85 ; 95 \% \mathrm{CI}$ : 1.85-3.86; $p<0.00001$ ) (Fig. 2).

Pyeloplasty. Three studies ${ }^{16,24,31}$ compared 2D and 3D laparoscopic systems during the pyeloplasty procedure (53 vs 45 patients, respectively). Of all the parameters reported in these studies, only operative time and EBL were comparable for meta-analysis. There was no significant difference between the two systems on both operative time and EBL (MD:
20.77; $95 \%$ CI: -24.82 to $66.35 ; p=0.37$ and MD: 10.88 ; 95\% CI: -19.44 to $41.20 ; p=0.48$, respectively) (Fig. 3).

Radical prostatectomy. Six studies ${ }^{18,19,21,28-30}$ compared 2D and 3D laparoscopic systems (containing 268 and 223 patients, respectively) during RPRP. Operative time and EBL revealed significant statistical differences between $2 \mathrm{D}$ and $3 \mathrm{D}$ laparoscopic imaging systems (MD: 42.85; 95\% CI: 17.44 68.27; $p<0.0001$ and MD: 78.38; 95\% CI: 31.59-125.1; $p=0.001$, respectively). Three-month postoperative continence recovery was significantly higher in the 3D laparoscopic radical prostatectomy (LRP) group than those operated on with 2D laparoscopy (OR: 0.40; 95\% CI: $0.21-0.78 ; p=0.007$ ). UVA time, PSMs, and hospital stays showed no statistical difference in the analysis (MD: 18.23 ; $95 \%$ CI: -1.78 to $38.24 ; p=0.07$, OR: 1.57 ; 95\% CI: $0.68-3.64 ; p=0.29$, and MD: $2.94 ; 95 \%$ CI: -2.20 to $8.90 ; p=0.26$, respectively) (Figs. 4 and 5).

\section{Discussion}

Several clinical and nonclinical studies have shown higher advantages in the 3D laparoscopic system over conventional 2D laparoscopy. ${ }^{5,9,33,34}$ Reduced performance time and lower precision errors favoring the 3D laparoscopic system are examples. ${ }^{35,36}$ Despite the advantages, 3D laparoscopy is not widely adopted. According to some authors, higher cost to purchase the $3 \mathrm{D}$ system compared with the $2 \mathrm{D}$ system is the main reason for its lack of broad adaptation. ${ }^{37}$ In contrast, Vettoretto and colleagues reported that adaption of the 3D system saves money for hospitals based on reduction in the operating time. ${ }^{38}$ However, other investigators failed to find any significant difference between $2 \mathrm{D}$ and $3 \mathrm{D}$ laparoscopic surgeries concerning total surgical expenses. ${ }^{27,28}$ On the other hand, discomfort, nausea, dizziness, and eye strains are among the reported limitations associated with the old generation of the 3D laparoscopic system. ${ }^{39}$ However, the new 3D high-definition generations did not show any of the above limitations compared with the 2D laparoscopic system. ${ }^{40}$

Urologists, in particular, showed special interest toward advances in 3D laparoscopic technology since most urological diseases can be managed with the assistance of laparoscopy. Many clinical studies compared 3D laparoscopy with the traditional 2D laparoscopic system during different urological surgeries to investigate the superiority claims favoring 3D laparoscopy. In this meta-analysis, data of 997 patients (548 vs 449 patients, 2D vs 3D laparoscopy, respectively) from 13 studies (3 randomized and 10 observational) were analyzed to evaluate the safety and efficacy of the 3D laparoscopic system compared with the 2D laparoscopic system.

\section{Partial nephrectomy}

In the past three decades, the role of PN in renal tumors has tremendously increased. Both European Association of Urology 2018 and American Urological Association 2017 guidelines on renal tumors strongly recommended PN as the standard treatment for localized T1 tumors. ${ }^{41,42}$ Although open, laparoscopic, and robotic approaches were equally suggested, minimally invasive techniques (laparoscopy or robotics) are preferred for their lower morbidity. ${ }^{42}$ Supporting these guidelines, a recent study with an extended follow-up period by Cai and colleagues ${ }^{43}$ compared laparoscopic partial nephrectomy (LPN) with laparoscopic radical nephrectomy 


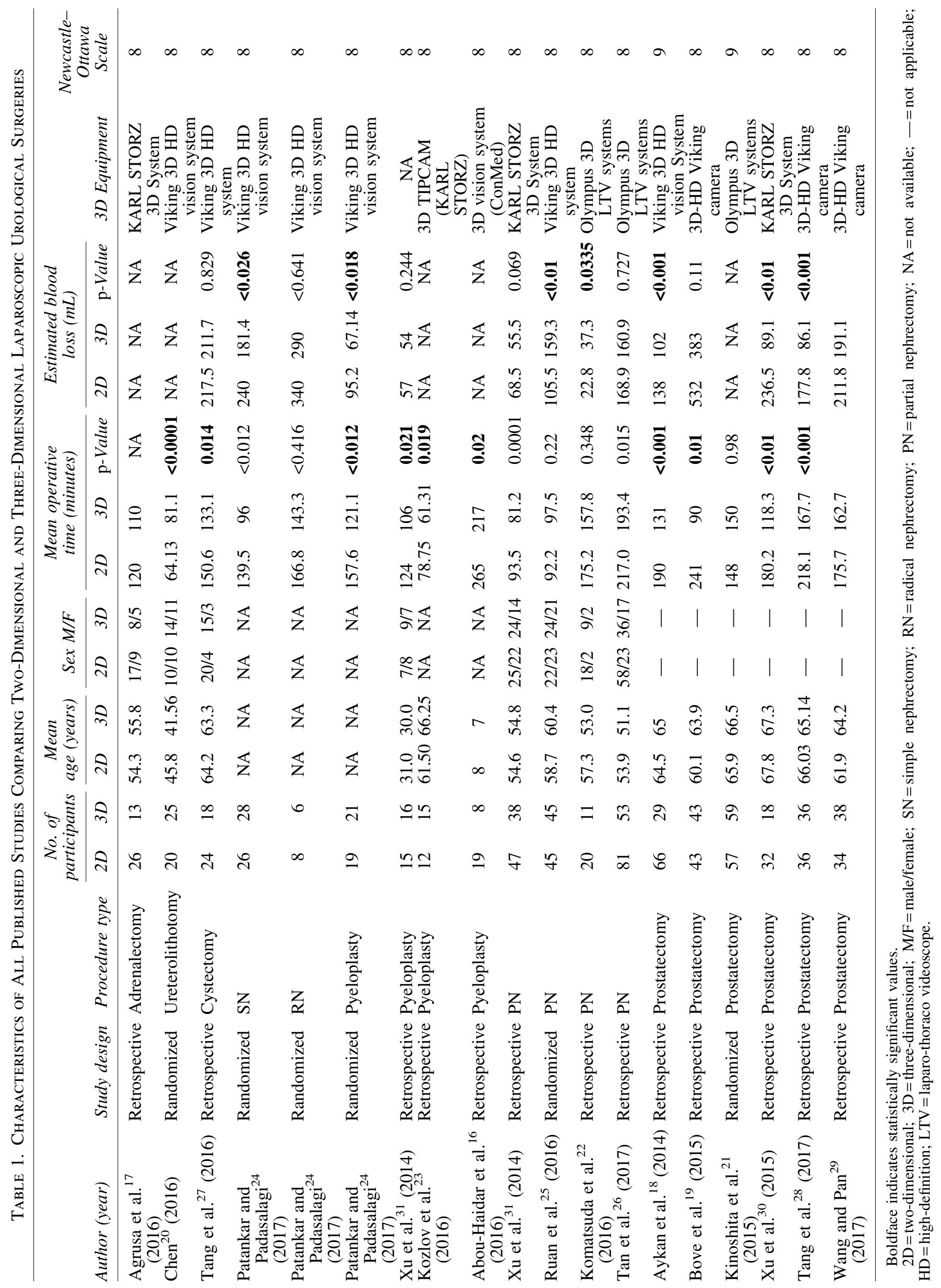


Table 2. Meta-Analysis Summary (Two-Dimensional vs Three-Dimensional Laparoscopic Systems in Urological Surgeries)

\begin{tabular}{|c|c|c|c|c|c|c|c|c|}
\hline \multirow[b]{2}{*}{ Outcome of interest } & \multirow{2}{*}{$\begin{array}{l}\text { No. of } \\
\text { studies }\end{array}$} & \multirow{2}{*}{$\begin{array}{l}\text { No. patients } \\
2 D \text { vs } 3 D\end{array}$} & \multirow[b]{2}{*}{$M D(95 \% C I)$} & \multirow[b]{2}{*}{$\mathrm{p}$} & \multicolumn{4}{|c|}{ Study heterogeneity } \\
\hline & & & & & $\chi^{2}$ test & $d f$ & $I^{2}(\%)$ & $\mathrm{p}$ \\
\hline \multicolumn{9}{|l|}{ Partial nephrectomy } \\
\hline Operative time (minutes) & 4 & $193 / 147$ & $9.56(-4.60$ to 23.73$)$ & 0.19 & 21.74 & 3 & 86 & 0.0001 \\
\hline $\mathrm{EBL}(\mathrm{mL})$ & 4 & $193 / 147$ & $-12.76(-50.56$ to 25.04$)$ & 0.51 & 30.79 & 3 & 90 & 0.0001 \\
\hline Dissecting time (minutes) & 2 & $65 / 56$ & $0.88(-4.02$ to 2.25$)$ & 0.58 & 5.19 & 1 & 81 & 0.02 \\
\hline Suturing time $(\mathrm{mL})$ & 2 & $65 / 56$ & $2.17(-2.19$ to 7.16$)$ & 0.28 & 20.30 & 1 & 95 & $<0.00001$ \\
\hline WIT (minutes) & 4 & $193 / 147$ & $2.85(1.85-3.86)$ & $<0.00001$ & 1.45 & 3 & 0 & 0.070 \\
\hline Pyeloplasty & & & & & & & & \\
\hline Operative time (minutes) & 3 & $53 / 45$ & $20.77(-24.82$ to 66.35$)$ & 0.37 & 22.82 & 2 & 91 & $<0.0001$ \\
\hline EBL $(\mathrm{mL})$ & 2 & $34 / 37$ & $10.88(-19.44$ to 41.20$)$ & 0.48 & 5.95 & 1 & 83 & 0.01 \\
\hline \multicolumn{9}{|l|}{ Prostatectomy } \\
\hline Operative time (minutes) & 6 & $268 / 223$ & $42.85(17.44-68.27)$ & $<0.001$ & 108.0 & 5 & 95 & $<0.0001$ \\
\hline $\mathrm{EBL}(\mathrm{mL})$ & 5 & $211 / 164$ & $78.38(31.59-125.1)$ & 0.001 & 74.65 & 4 & 95 & $<0.0001$ \\
\hline UVA (minutes) & 4 & 206/169 & $18.23(-1.78$ to 38.24$)$ & 0.07 & 574.3 & 3 & 99 & $<0.0001$ \\
\hline Hospital stay (days) & 2 & $68 / 54$ & $2.94(-2.20$ to 8.90$)$ & 0.26 & 5.25 & 1 & 98 & 0.02 \\
\hline PSM & 4 & $177 / 126$ & OR: $1.57(0.68-3.64)$ & 0.29 & 0.26 & 2 & 0 & 0.88 \\
\hline Continence recovery & 4 & $177 / 145$ & OR: $0.40(0.21-0.78)$ & 0.007 & 0.13 & 3 & 27 & 0.25 \\
\hline
\end{tabular}

Boldface indicates statistically significant values.

$\mathrm{WIT}=$ warm ischemia time; $\mathrm{UVA}=$ urethrovesical anastomosis; $\mathrm{MD}=$ mean difference; $\mathrm{OR}=$ odds ratio; $\mathrm{CI}=$ confidence interval; $\mathrm{EBL}=$ estimated blood loss; PSM = positive surgical margin.

(LRN). In the above study, they found $85.56 \%$ vs $85.69 \%$ and $88.00 \%$ vs $82.85 \%$ of 10 years overall and cancer-specific survival rates after LPN and LRN, respectively. These results indicate that LPN is equally effective as LRN in terms of cancer control in addition to its nephron-sparing advantage.
LPN is one of the commonly performed urological surgeries today. Since several nonclinical studies reported shorter suturing time in the 3D laparoscopic system, we expected similar results in our meta-analysis. However, only WIT was significantly shorter during 3D laparoscopic

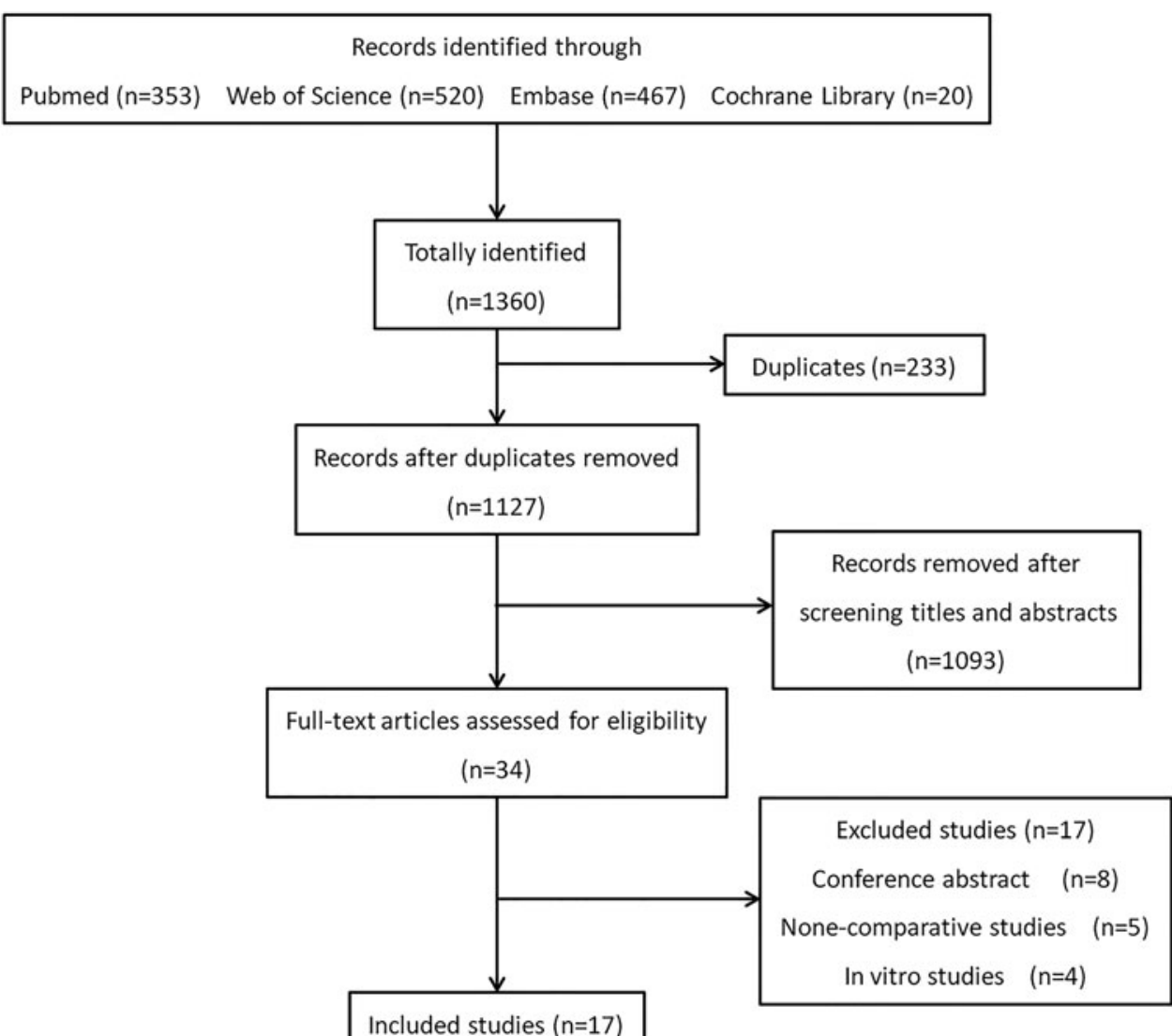

FIG. 1. Flow chart of study search, inclusions, and exclusions. 


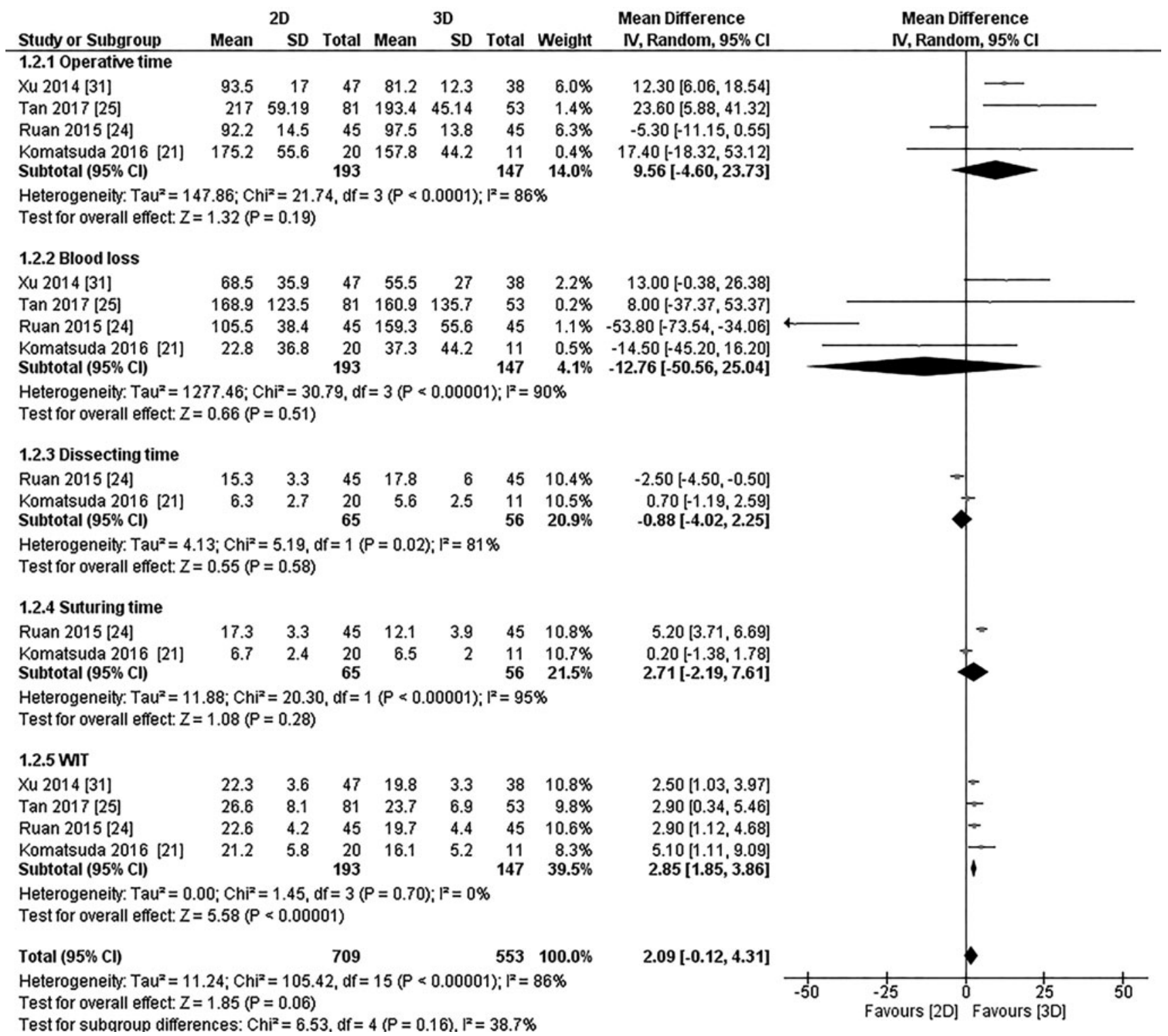

FIG. 2. Forest plot and meta-analysis results of partial nephrectomy (2D vs 3D laparoscopy). 2D=two-dimensional; $3 \mathrm{D}=$ three-dimensional; $\mathrm{M}-\mathrm{H}=$ Mantel-Haenszel; $\mathrm{CI}=$ confidence interval.

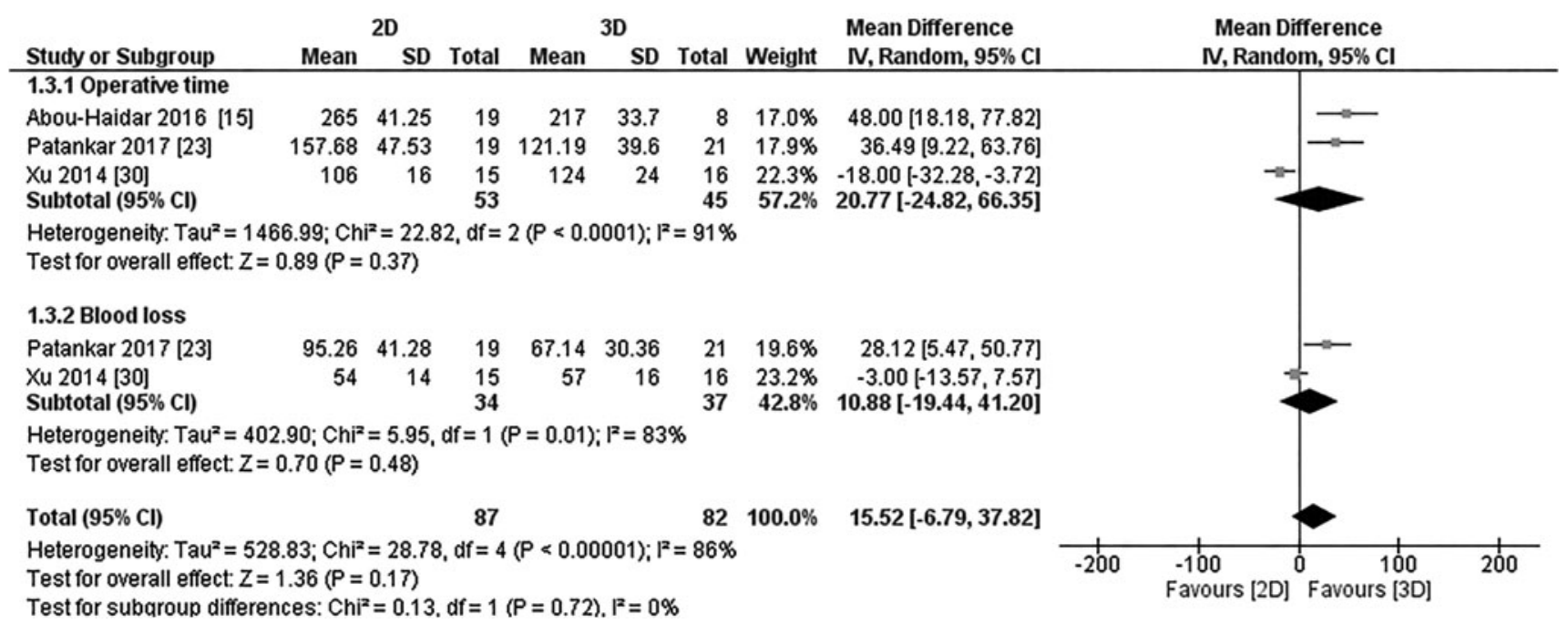

FIG. 3. Forest plot and meta-analysis results of the pyeloplasty procedure (2D vs 3D laparoscopy). 


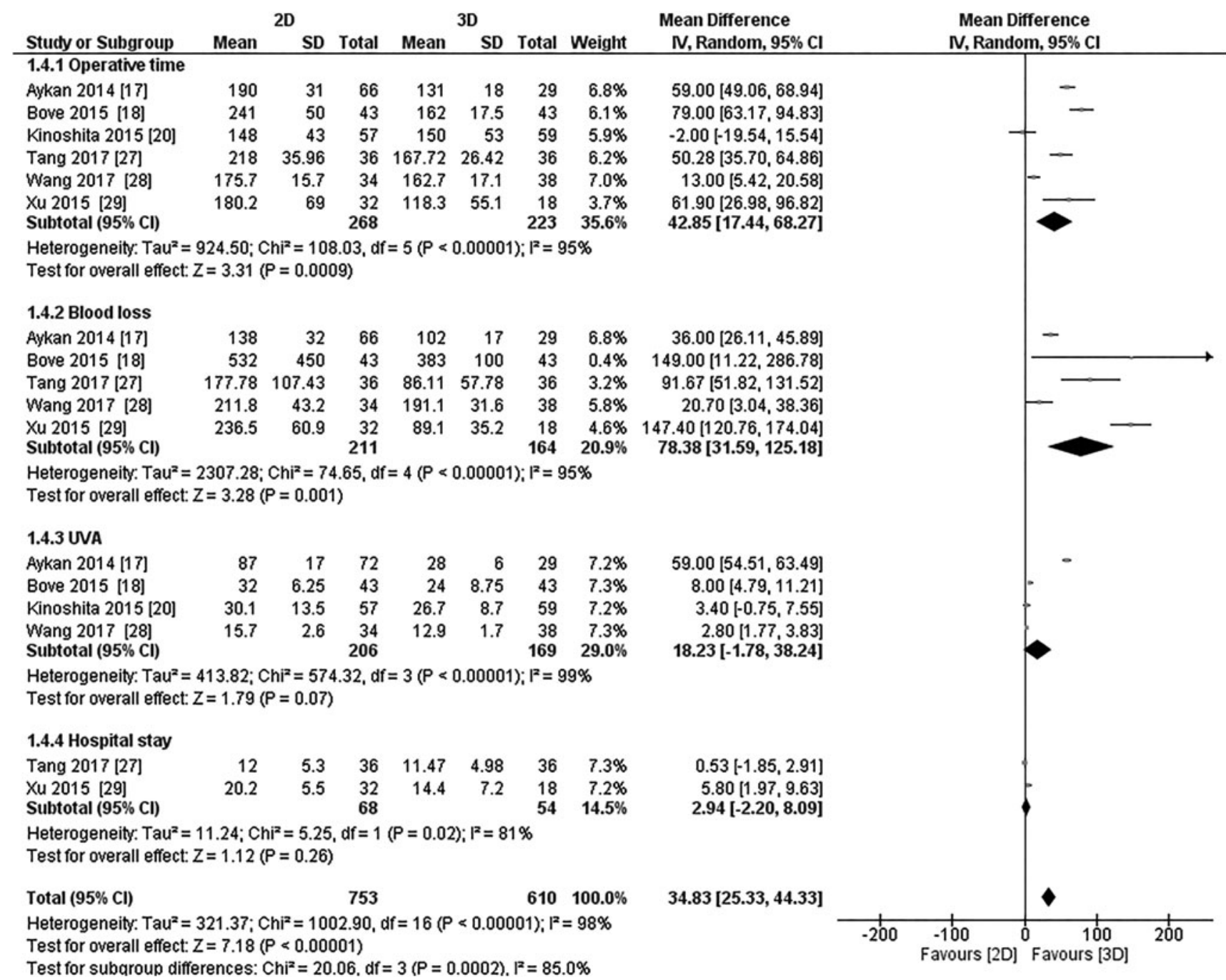

FIG. 4. Forest plot and meta-analysis results of radical prostatectomy (2D vs 3D laparoscopy).

surgery, while other parameters such as operative time, EBL, dissecting time, and suturing time were comparable between the 2D and 3D laparoscopic systems during PN.

On the other hand, we should keep in mind that there are other factors such as surgeon's experience, operative approach, and R.E.N.A.L nephrometry score (RNS) that can influence surgical and clinical outcomes during LPN. Different operative approaches have different perioperative outcomes; for instance, a meta-analysis study by Fan et al. ${ }^{44}$ reported higher operative time and more extended hospital stays in transperitoneal LPN (TLPN) compared with retroperitoneal LPN (RLPN), while WIT and EBL were not statistically different between TLPN and RLPN. Of the four studies ${ }^{22,25,26,32}$ in our meta-analysis, only Komatsuda and colleagues ${ }^{22}$ approached their cases transperitoneally, while the other three authors reported a retroperitoneal approach.

According to some reports, higher RNS is associated with more extended WIT and higher conversion rates (to open) during LPN. ${ }^{45}$ Nevertheless, two of the three studies in our meta-analysis reported results of RNS with no significant difference between the groups.

\section{Pyeloplasty}

Laparoscopic pyeloplasty is a widely accepted procedure for management of ureteropelvic junction obstruction. Both transperitoneal laparoscopic pyeloplasty (TLP) and retroperitoneal laparoscopic pyeloplasty (RLP) approaches are safe and effective. However, according to Singh et al., ${ }^{46}$ higher operative time and intracorporeal suturing time were found in RLP compared with TLP. Another study found that mean drainage output $(\mathrm{mL})$ was significantly more in continuous sutured TLP compared with interrupted sutured TLP. ${ }^{47}$

In this study, we considered pyeloureteral intracorporeal suturing time as a crucial parameter. Unfortunately, none of the studies we reviewed have provided any data regarding this vital parameter for comparison. Two of the three studies $^{16,31}$ in our meta-analysis comparing $2 \mathrm{D}$ with $3 \mathrm{D}$ laparoscopic pyeloplasty showed significantly shorter operative time favoring the 3D laparoscopic system. However, our meta-analysis failed to show any significant difference between 2D and 3D laparoscopic pyeloplasty concerning operative time and EBL. 


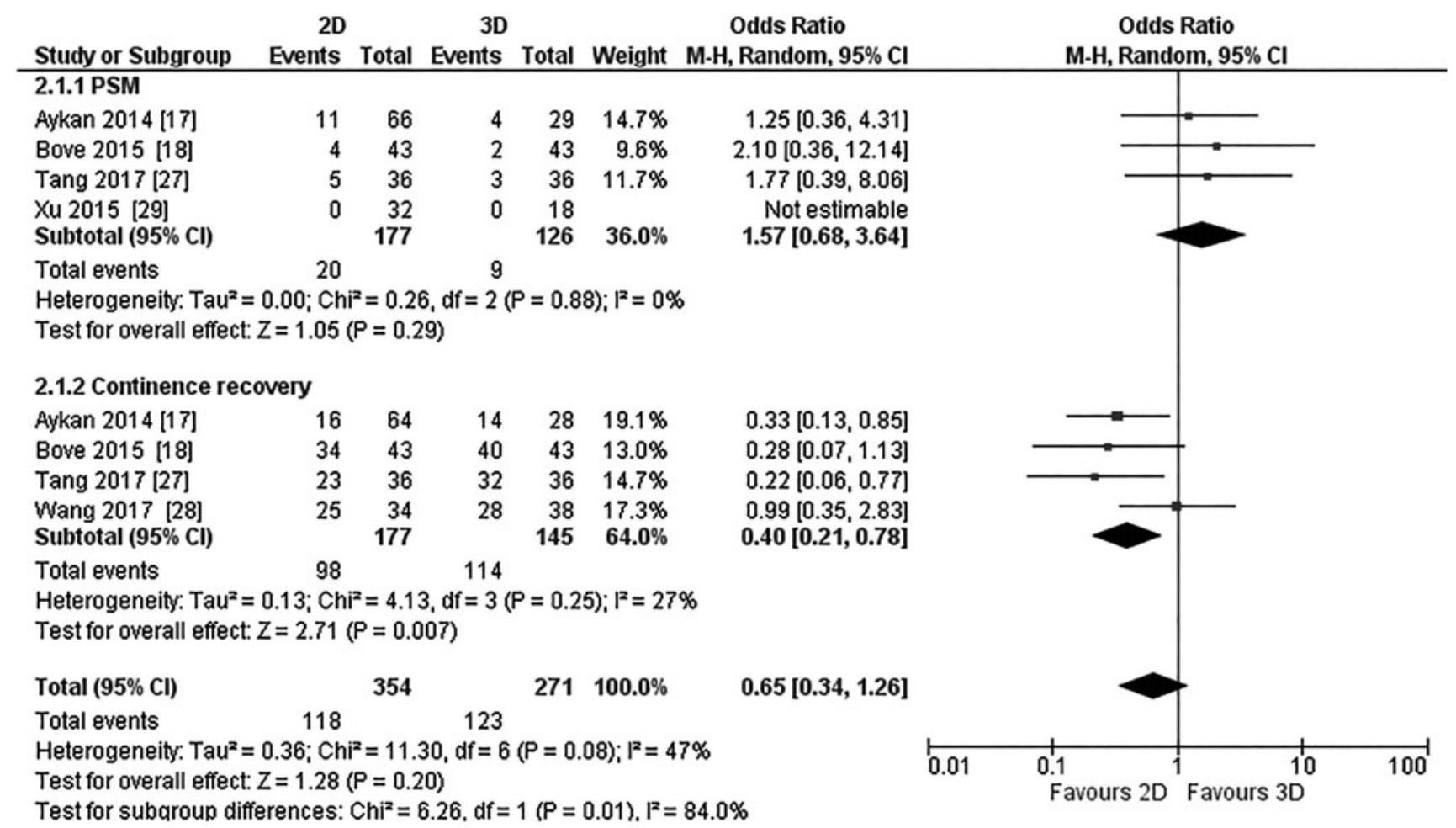

FIG. 5. Forest plot and meta-analysis results of PSMs and continence recovery after radical prostatectomy (2D $v s 3 \mathrm{D}$ laparoscopy). PSMs = positive surgical margins.

\section{Radical prostatectomy}

Prostate cancer is the second most diagnosed cancer in men after lung cancer. ${ }^{48}$ Perhaps this is due to the increase in human life expectancy (since prostate cancer is an age-related disease) and the extensive use of screening programs containing prostate-specific antigen examination and high-quality imaging techniques. Although open radical prostatectomy (ORP) is still the golden approach, almost all medium- to large-sized urological centers around the world are well adapted with LRP or robot-assisted radical prostatectomy (RARP) techniques for their lower morbidity. Irrespective of the method used during $\mathrm{RP}$, five common goals should be achieved after the surgery, the so-called pentafecta (no evidence of PSMs, no early complications, sexual and continence recovery, and free from biochemical recurrence). ${ }^{49} \mathrm{~A}$ newly published review article by Basiri et al. ${ }^{2}$ reported higher PSM and complication rates in ORP than in RARP. In the same study, operative time, length of hospital stay, EBL, and transfusion rates were significantly lower in LRP than in ORP.

$\mathrm{RP}$ is a complex and delicate procedure that requires detailed anatomical understanding, especially when dealing with young patients with localized tumors, for better oncological and functional outcomes. For this reason, many centers around the world use the da Vinci robotic system for its excellent 3D vision and hand-like EndoWrist instrument. According to the Intuitive Surgical, Inc., 2016 annual report, over 162,000 RARP procedures were performed during 2016 alone. ${ }^{50}$ Nevertheless, the use of the da Vinci robotic machine costs much money for both patients and healthcare providers. ${ }^{2}$ Therefore, advances in traditional laparoscopy such as 3D technology and robotized laparoscopic instruments are essential for their lower cost compared with da Vinci robotics.

The operative time and EBL were significantly shorter in 3D LRP than in 2D laparoscopy in our meta-analysis, while PSMs and length of hospital stay were similar between the two groups. On the other hand, we might all agree that UVA during LRP requires complex maneuvers to accomplish leak-free anastomosis. The angle at which the anastomosis should be performed plus the limited motion of laparoscopic instruments makes UVA the most challenging step in the whole procedure. Of the four studies that reported UVA time, three of them ${ }^{18,19,29}$ found significantly higher UVA time in 2D laparoscopy compared with the 3D laparoscopic system. Nevertheless, this meta-analysis did not notice any statistical significance between the 2D and 3D laparoscopic systems regarding UVA time $(p=0.07)$. Similarly, PSM rates were not significantly different between the two groups. In contrast, 3-month postprostatectomy continence recovery was higher in the 3D laparoscopic group $(p=0.007)$, perhaps the 3D laparoscopic imaging system improves visibility to allow preservation of important structures for faster continence recovery.

Sexual recovery after RP is another vital surgical outcome. Only two studies reported potency recovery outcomes in which results were conflicting. Bove and colleagues ${ }^{19}$ reported similar potency outcomes (58\% vs 63\% in 2D and 3D laparoscopy, respectively) after bilateral nerve-sparing surgery. On the other hand, Tang et al. ${ }^{28}$ found significantly faster sexual recovery in 3D LRP than in the 2D operated group (41.67\% vs $25 \%$ and $58.33 \%$ vs $41.67 \%$ after 3 and 6 months, respectively).

The small data sample in our meta-analysis and shortcomings of observational studies are the two main limitations of our study; these two together may prevent an absolute conclusion. 


\section{Conclusion}

Although the data sample is small for a concrete and conclusive argument, based on the above findings in our meta-analysis, the 3D laparoscopic imaging system seems to provide better clinical and surgical outcomes in some urological procedures compared with conventional 2D laparoscopy. Three-dimensional laparoscopy mainly improves the depth of perception for better visibility, which is important for complex urological surgeries such as PN, pyeloplasty, and RP. Future well-designed, randomized clinical studies comparing 2D and 3D laparoscopic systems are recommended to validate our findings.

\section{Acknowledgment}

The authors are grateful to Dr. Musa Male for grammatical corrections in the manuscript.

\section{Author Disclosure Statement}

No competing financial interests exist.

\section{References}

1. Tarasconi JC. Endoscopic salpingectomy. J Reprod Med 1981;26:541-545.

2. Basiri A, de la Rosette JJ, Tabatabaei S, Woo HH, Laguna MP, Shemshaki H. Comparison of retropubic, laparoscopic and robotic radical prostatectomy: Who is the winner? World J Urol 2018;36:609-621.

3. Eskicorapci SY, Teber D, Schulze M, Ates M, Stock C, Rassweiler JJ. Laparoscopic radical nephrectomy: The new gold standard surgical treatment for localized renal cell carcinoma. ScientificWorldJournal 2007;7:825-836.

4. Criss CN, Ralls MW, Johnson KN, Awtar S, Jarboe MD, Geiger JD. A novel intuitively controlled articulating instrument for reoperative foregut surgery: A case report. J Laparoendosc Adv Surg Tech A 2017;27:983-986.

5. Wenzl R, Pateisky N, Husslein P. First use of a 3D videoendoscope in gynecology. Geburtshilfe Frauenheilkd 1993; 53:776-778.

6. Sinha R, Sundaram M, Raje S, Rao G, Sinha M, Sinha R. 3D laparoscopy: Technique and initial experience in 451 cases. Gynecol Surg 2013;10:123-128.

7. van Beurden MHPH, IJsselsteijn WA, Juola JF. Effectiveness of stereoscopic displays in medicine: A review. 3D Res 2012;3:3.

8. Sahu D, Mathew MJ, Reddy PK. 3D laparoscopy-help or hype; initial experience of a tertiary health centre. J Clin Diagn Res 2014;8:NC01-NC03.

9. Cicione A, Autorino R, Breda A, et al. Three-dimensional vs standard laparoscopy: Comparative assessment using a validated program for laparoscopic urologic skills. Urology 2013;82:1444-1450.

10. Cicione A, Autorino R, Laguna MP, et al. Three-dimensional technology facilitates surgical performance of novice laparoscopy surgeons: A quantitative assessment on a porcine kidney model. Urology 2015;85:1252-1256.

11. He B, Mou L, De Roo R, Musk GC, Hamdorf JM. Evaluation of three-dimensional versus conventional laparoscopy for kidney transplant procedures in a human cadaveric model. Exp Clin Transplant 2017;15:497-503.

12. Leon P, Rivellini R, Giudici F, Sciuto A, Pirozzi F, Corcione F. 3D vision provides shorter operative time and more accurate intraoperative surgical performance in laparoscopic hiatal hernia repair compared with 2D vision. Surg Innov 2017;24:155-161.

13. Komaei I, Navarra G, Currò G. Three-dimensional versus two-dimensional laparoscopic cholecystectomy: A systematic review. J Laparoendosc Adv Surg Tech A 2017;27:790-794.

14. Moher D, Shamseer L, Clarke M, et al. Preferred reporting items for systematic review and meta-analysis protocols (PRISMA-P) 2015 statement. Syst Rev 2015;4:1.

15. Hozo SP, Djulbegovic B, Hozo I. Estimating the mean and variance from the median, range, and the size of a sample. BMC Med Res Methodol 2005;5:13.

16. Abou-Haidar H, Al-Qaoud T, Jednak R, Brzezinski A, ElSherbiny M, Capolicchio J. Laparoscopic pyeloplasty: Initial experience with 3D vision laparoscopy and articulating shears. J Pediatr Urol 2016;12:426.e1-426.e5.

17. Agrusa A, di Buono G, Chianetta D, et al. Three-dimensional (3D) versus two-dimensional (2D) laparoscopic adrenalectomy: A case-control study. Int J Surg 2016;28(Suppl 1): S114-S117.

18. Aykan S, Singhal P, Nguyen DP, et al. Perioperative, pathologic, and early continence outcomes comparing three-dimensional and two-dimensional display systems for laparoscopic radical prostatectomy - a retrospective, single-surgeon study. J Endourol 2014;28:539-543.

19. Bove P, Iacovelli V, Celestino F, De Carlo F, Vespasiani G, Finazzi Agrò E. 3D vs 2D laparoscopic radical prostatectomy in organ-confined prostate cancer: Comparison of operative data and pentafecta rates: A single cohort study. BMC Urol 2015;15:12.

20. Chen Q. [Comparison of the effect of three-dimensional versus two-dimensional retroperitoneal laparoscopic ureter lithotomy]. Nan Fang Yi Ke Xue Xue Bao 2016;36:148150 (Article in Chinese).

21. Kinoshita H, Nakagawa K, Usui Y, et al. High-definition resolution three-dimensional imaging systems in laparoscopic radical prostatectomy: Randomized comparative study with high-definition resolution two-dimensional systems. Surg Endosc 2015;29:2203-2209.

22. Komatsuda A, Matsumoto K, Miyajima A, Kaneko G, Mizuno R, Kikuchi E, Oya M. Technical improvement using a three-dimensional video system for laparoscopic partial nephrectomy. Asian Pac J Cancer Prev 2016;17:2475-2478.

23. Kozlov Y, Kovalkov K, Nowogilov V. 3D laparoscopy in neonates and infants. J Laparoendosc Adv Surg Tech A 2016;26:1021-1027.

24. Patankar SB, Padasalagi GR. Three-dimensional versus two-dimensional laparoscopy in urology: A randomized study. Indian J Urol 2017;33:226-229.

25. Ruan Y, Wang XH, Wang K, Zhao YY, Xia SJ, Xu DL. Clinical evaluation and technical features of three-dimensional laparoscopic partial nephrectomy with selective segmental artery clamping. World J Urol 2016;34:679-685.

26. Tan H, Tang X, Wu Z, et al. Clinical comparative analysis of 3D and 2D laparoscopic partial nephrectomy for renal tumors. Acad J Second Mil Med Univ 2017;38:239-243.

27. Tang FJ, Qi L, Jiang HC, Tong SY, Li Y. Comparison of the clinical effectiveness of 3D and 2D imaging systems for laparoscopic radical cystectomy with pelvic lymph node dissection. J Int Med Res 2016;44:613-619.

28. Tang KQ. [Three-dimensional versus two-dimensional imaging systems in laparoscopic radical prostatectomy for prostate cancer: A retrospective cohort study]. Nan Fang Yi Ke Xue Xue Bao 2017;37:1-5 (Article in Chinese). 
29. Wang H, Pan T. Comparison of the postoperative recovery of urinary continence after 3D and 2D laparoscopic radical prostatectomy. Chin J Urol 2017;38:182-186.

30. Xu B, Liu N, Jiang $H$, et al. 3D versus 2D laparoscopic radical prostatectomy for the treatment of prostate cancer. Zhonghua Nan Ke Xue 2015;21:904-907 (Article in Chinese).

31. Xu W. Comparison of three dimensional and two dimensional laparoscopic pyeloplasty for ureteropelvic junction obstruction. Zhonghua Wai Ke Zhi 2014;52:771-774 (Article in Chinese).

32. Xu W, Li H, Zhang Y, Zhang X, Yan W, Ji Z, Xiao H, Liu G, Wen J, et al. Comparison of 3D and 2D laparoscopic nephron sparing surgery for renal tumor. Chin J Urol 2014; 35:410-413.

33. Schoenthaler M, Schnell D, Wilhelm K, Schlager D, Adams F, Hein S, Wetterauer U, Miernik A. Stereoscopic (3D) versus monoscopic (2D) laparoscopy: Comparative study of performance using advanced HD optical systems in a surgical simulator model. World J Urol 2016;34:471-477.

34. Lu J, Zheng CH, Zheng HL, et al. Randomized, controlled trial comparing clinical outcomes of 3D and 2D laparoscopic surgery for gastric cancer: An interim report. Surg Endosc 2017;31:2939-2945.

35. Sørensen SM, Savran MM, Konge L, Bjerrum F. Threedimensional versus two-dimensional vision in laparoscopy: A systematic review. Surg Endosc 2016;30:11-23.

36. Sørensen SMD, Konge L, Bjerrum F. 3D vision accelerates laparoscopic proficiency and skills are transferable to $2 \mathrm{D}$ conditions: A randomized trial. Am J Surg 2017;214:63-68.

37. Alaraimi B, El Bakbak W, Sarker S, et al. A randomized prospective study comparing acquisition of laparoscopic skills in three-dimensional (3D) vs. two-dimensional (2D) laparoscopy. World J Surg 2014;38:2746-2752.

38. Vettoretto N, Foglia E, Ferrario L, et al. Why laparoscopists may opt for three-dimensional view: A summary of the full HTA report on 3D versus 2D laparoscopy by S.I.C.E. (Societa Italiana di Chirurgia Endoscopica e Nuove Tecnologie). Surg Endosc 2018;32:2986-2993.

39. Wilhelm D, Reiser S, Kohn N, et al. Comparative evaluation of HD 2D/3D laparoscopic monitors and benchmarking to a theoretically ideal 3D pseudodisplay: Even well-experienced laparoscopists perform better with 3D. Surg Endosc 2014;28: 2387-2397.

40. Usta TA, Ozkaynak A, Kovalak E, Ergul E, Naki MM, Kaya E. An assessment of the new generation three-dimensional high definition laparoscopic vision system on surgical skills: A randomized prospective study. Surg Endosc 2015;29:2305-2313.

41. Campbell S, Uzzo RG, Allaf ME, et al. Renal mass and localized renal cancer: AUA Guideline. J Urol 2017;198:520-529.

42. Ljungberg B, Bensalah K, Bex A, et al. Renal Cell Carcinoma. EAU Guidelines Presented at EAU Annual Congress Copenhagen 2018. ISBN 978-94-92671-01-1, 2018. EAU Guidelines Office, Arnhem, The Netherlands. http://uroweb .org/guidelines/compilations-of-all-guidelines/

43. Cai Y, Li HZ, Zhang YS. Comparison of partial and radical laparascopic nephrectomy: Long-term outcomes for clinical T1b renal cell carcinoma. Urol J 2018;15:16-20.

44. Fan X, Xu K, Lin T, Liu H, Yin Z, Dong W, Huang H, Huang J. Comparison of transperitoneal and retroperitoneal laparoscopic nephrectomy for renal cell carcinoma: A systematic review and meta-analysis. BJU Int 2013;111:611-621.

45. Mayer WA, Godoy G, Choi JM, Goh AC, Bian SX, Link RE. Higher RENAL nephrometry score is predictive of longer warm ischemia time and collecting system entry during lap- aroscopic and robotic-assisted partial nephrectomy. Urology 2012;79:1052-1056.

46. Singh V, Sinha RJ, Gupta DK, Kumar V, Pandey M, Akhtar A. Prospective randomized comparison between transperitoneal laparoscopic pyeloplasty and retroperitoneoscopic pyeloplasty for primary ureteropelvic junction obstruction. JSLS 2014;18:pii:e2014.00366.

47. Ramalingam M, Kallappan S, Nachimuthu S. A prospective comparative study of continuous and interrupted suturing in laparoscopic pyeloplasty in 3D era. J Laparoendosc Adv Surg Tech A 2018; [Epub ahead of print]; DOI: 10.1089/ lap.2018.0203.

48. Ferlay J, Soerjomataram I, Dikshit R, et al. Cancer incidence and mortality worldwide: Sources, methods and major patterns in GLOBOCAN 2012. Int J Cancer 2015; 136:E359-E386.

49. Patel VR, Abdul-Muhsin HM, Schatloff O, et al. Critical review of 'pentafecta' outcomes after robot-assisted laparoscopic prostatectomy in high-volume centres. BJU Int 2011;108:1007-1017.

50. Intuitive Surgical I. Annu Rep 2016. 2016. Available at: www.intuitivesurgical.com (last accessed May 18, 2018).

Address correspondence to:

Shaogang Wang, PhD

Department of Urology

Tongji Hospital

Tongji Medical College

Huazhong University of Science and Technology Liberalization Avenue, No. 1095

Wuhan 430030

P.R. China

E-mail: sgwangtjm@163.com

$$
\begin{aligned}
& \text { Abbreviations Used } \\
2 \mathrm{D} & =\text { two-dimensional } \\
3 \mathrm{D} & =\text { three-dimensional } \\
\mathrm{CI} & =\text { confidence interval } \\
\mathrm{EBL} & =\text { estimated blood loss } \\
\mathrm{HD} & =\text { high-definition } \\
\mathrm{LPN} & =\text { laparoscopic partial nephrectomy } \\
\mathrm{LRN} & =\text { laparoscopic radical nephrectomy } \\
\mathrm{LRP} & =\text { laparoscopic radical prostatectomy } \\
\mathrm{LTV} & =\text { laparo-thoraco videoscope } \\
\mathrm{MD} & =\text { mean difference } \\
\mathrm{NA} & =\text { not available } \\
\mathrm{OR} & =\text { odds ratio } \\
\mathrm{ORP} & =\text { open radical prostatectomy } \\
\mathrm{PN} & =\text { partial nephrectomy } \\
\mathrm{PSMs} & =\text { positive surgical margins } \\
\mathrm{RARP} & =\text { robot-assisted radical prostatectomy } \\
\mathrm{RLP} & =\text { retroperitoneal laparoscopic pyeloplasty } \\
\mathrm{RLPN} & =\text { retroperitoneal laparoscopic partial } \\
\mathrm{RN} & =\text { nephrectomy } \\
\mathrm{RNS} & =\mathrm{R} . \mathrm{E} . \mathrm{N} . \text { A.L } . \mathrm{L} \text { nephrometry score } \\
\mathrm{RP} & =\text { radical prostatectomy } \\
\mathrm{SN} & =\text { simple nephrectomy } \\
\mathrm{TLP} & =\text { transperitoneal laparoscopic } \\
\text { TLPN } & =\text { transperitoneal laparoscopic partial } \\
\mathrm{UVA} & =\text { nephrectomy } \\
\mathrm{WIT} & =\text { warm ischemia time }
\end{aligned}
$$

